\title{
A Case of Erythema Nodosum and Serositis Associated with Myelodysplastic Syndrome
}

\author{
Jung-Hye Choi, M.D., Myung-Ju Ahn, M.D., Yong-Wook Park, M.D. ${ }^{2}$, \\ Ho-Suk Oh, M.D., Young-Yeul Lee, M.D. and In-Soon Kim, M.D. \\ Departments of Internal Medicine and Pathology?, \\ Hanyang University College of Medicine, Seoul, Korea
}

\begin{abstract}
Myelodysplastic syndrome (MDS) is a heterogenous group of stem cell disorders usually characterized by progressive refractory cytopenias, which could progress to acute myeloid leukemia. MDS may be associated with a wide spectrum of skin lesions, including neoplastic cell infiltration, Sweet's syndrome, pyoderma gangrenosum, erythema elevatum diutinum, vasculitis, and panniculitis. However, erythema nodosum is rarely associated with MDS. Unusual rheumatologic manifestations in patients with MDS also have been reported, which range from asymptomatic serological abnormalities to classic connective tissue disorders such as Sjögren's syndrome, relapsing polychondritis, systemic lupus erythematosus, rheumatoid arthritis and mixed connective tissue disease. However, concurrent erythema nodosum and serositis has rarely been reported. We describe a case of MDS with erythema nodosum and immune-mediated pericardial effusion in a 34-year-old woman.
\end{abstract}

Key Words : Myelodysplastic syndrome, Erythema nodosum, Pericardial effusion

\section{INTRODUCTION}

The myelodysplastic syndrome (MDS) is a heterogenous group of stem cell disorders usually characterized by progressive refractory cytopenias with possible progression to acute myeloid leukemia. Although cutaneous manifestation in MDS is not frequent, MDS may be associated with a wide spectrum of skin lesions ${ }^{11}$ and unusual rheumatologic manifestations ${ }^{2,3)}$. However, concurrent erythema nodosum and serositis has rarely been reported. We describe a case of MDS with erythema nodosum and immune- mediated pericardial effusion.

\section{CASE REPORT}

A 34-year-old woman was diagnosed with MDS FrenchAmerican-British (FAB) subtype refractory anemia in March, 2000. The patient did not receive any special treatment except intermittent transfusion of packed red cell.

In May, 2003 the patient presented with fatigue, myalgia, fever as well as multiple edematous, tender, erythematous to brownish subcutaneous nodular lesions on both upper and lower extremities and trunk (Figure 1). Laboratory finding indicated hemoglobin $5.8 \mathrm{~g} / \mathrm{dL}$, white blood cell count $6.8 \times$ $10^{9} / \mathrm{L}$, platelet $4.8 \times 10^{10} / \mathrm{L}$, and normal liver and kidney function test. Biopsy of skin lesion revealed lobular panniculitis consistent with erythema nodosum (Figure 2). The skin lesion had improved with $20 \mathrm{mg}$ of prednisone per day.

One month later, dyspnea, chest pain and fever developed and the skin lesions were aggravated. She was taking a tapering dose of prednisone $(10 \mathrm{mg})$. The laboratory tests showed hemoglobin $6.0 \mathrm{~g} / \mathrm{dL}$, white blood cell count $4.8 \times 10^{9} / \mathrm{L}$, platelet $8.0 \times 10^{9} / \mathrm{L}$, uric acid $2.3 \mathrm{mg} / \mathrm{dL}, \mathrm{LDH} 474 \mathrm{U} / \mathrm{L}$ (normal 211-423), serum ferritin $1831 \mathrm{ng} / \mathrm{mL}$, serum iron/TIBC 145/162 $\mu$ g/dL. Antinuclear antibody, rheumatoid factor and ANCA were all negative. The levels of C3 and C4 were normal. Blood and

- Received : August 24, 2004

- Accepted : November 18, 2004

- Correspondence to : Jung-Hye Choi M.D., Department of Internal Medicine, Hanyang University Guri Hospital, 249-1, Gyomoon-dong, Guri city, Gyunggi-do, 471-701, Korea TEL : 82-31-560-2236, FAX : 82-31-553-7369, Email : jhcmd@ hanyang.ac.kr 


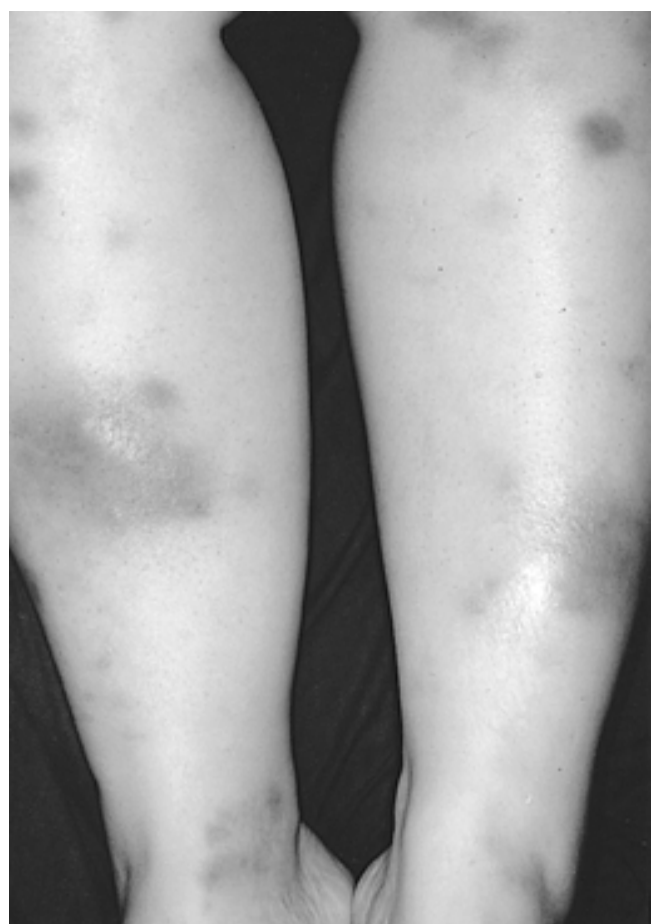

Figure 1. Multiple edematous, tender, erythematous nodules on the extensor surfaces of the legs.

urine culture were sterile. Chest X-ray and chest CT scan showed cardiomegaly with pulmonary edema (Figure 3). Echocardiography revealed normal ejection fraction with large amounts of pericardial effusion. After the dose of prednisone was increased to $40 \mathrm{mg} /$ day, the pericardial effusion and skin lesions had remarkably improved, suggesting that these newly developed symptoms might be attributed to the immune-mediated complications of MDS. The repeated bone marrow biopsy performed one month later revealed no evidence of progression to acute leukemia. However, the dysplastic changes in the myeloid, erythroid and megakaryocytes lineages had progressed compared to the examination in the prior month. While gradually tapering prednisone until July 2004, she was taking $5 \mathrm{mg} /$ day of prednisone without pericardial effusion. Platelet count slowly increased and maintained above $2.0 \times 10^{10} / \mathrm{L}$.

\section{DISCUSSION}

MDS can be associated with a wide spectrum of skin lesions including neoplastic cell infiltration, Sweet's syndrome, pyoderma gangrenosum, erythema elevatum diutinum, vasculitis, and panniculitis ${ }^{11}$. However, MDS-associated erythema nodosum is relatively rare ${ }^{4)}$. Erythema nodosum is the most frequent clinico-pathological variant of panniculitis and manifests as a cutaneous reaction consisting of inflammatory, tender, nodular

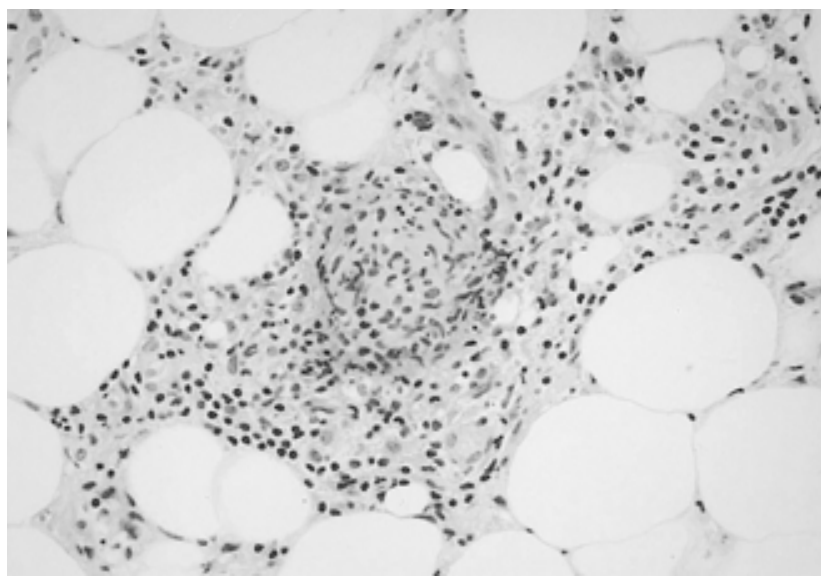

Figure 2. Histological examination showed lympho-histiocytic infiltration in the septa and the periphery of the fat lobules consistent with lobular panniculitis (H\&E stain, $\times 400)$.

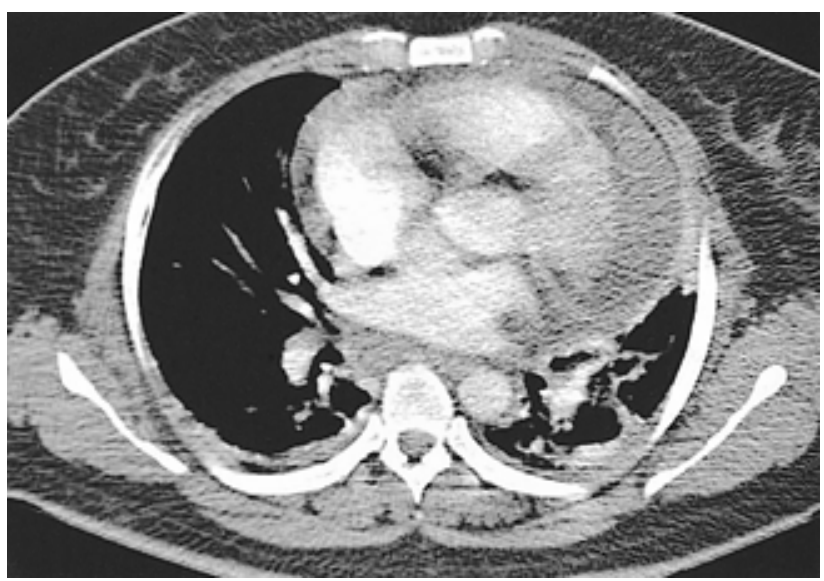

Figure 3. Chest CT showed pericardial effusion with pulmonary edema and atelectasis.

lesions, usually located symmetrically on the extensor surfaces of the lower extremities ${ }^{5)}$. Manifestation of the lesions may be associated with a wide variety of conditions including infections, sarcoidosis, rheumatologic diseases, inflammatory bowel diseases, medications, autoimmune disorders, pregnancy and malignancies $^{6-8)}$. The appearance of skin lesions in MDS may herald its progression to acute myeloid leukemia. Although in this case the repeated bone marrow biopsy did not show any findings of progression to acute leukemia, the dysplastic changes in all lineages of hematopoietic cells had progressed with the development of skin lesions.

It has been reported that autoimmune manifestations in patients with MDS range from asymptomatic serological abnormalities to classic connective tissue disorders such as Sjögren's syndrome, relapsing polychondritis, systemic lupus erythematosus, rheumatoid arthritis and mixed connective tissue 
disease $e^{2,3,9)}$.

The pathogenesis of autoimmune features in MDS has not been determined. Several reports describe the dysregulation of cellular and humoral immunity. In particular, abnormal T-cell responses to antigen presentation and abnormal $\mathrm{B}-$ cell and T-cell interactions in MDS may be important in the pathogenesis of immune dysregulation leading to the development of an autoimmune phenomenon ${ }^{10-12)}$. Alternatively, autoimmune manifestations may precede the onset of MDS. Thus, abnormalities in T-cell function might be associated with the development of $\mathrm{MDS}^{13}$. Although pericardiocentesis was not performed in our case, the pericardial effusion rapidly regressed after treatment with high doses of systemic corticosteroid, suggesting that this lesion might be a reactive inflammatory change involving immunologic mechanisms.

Enright et al. $^{9)}$ reported that aggressive therapy with immunosuppressive agents in selected patients often controls autoimmune features associated with MDS and may lead to hematological responses in some patients. The most commonly used immunosuppressive agents are oral prednisone and high-dose intravenous methylprednisolone. In addition, some patients received azathioprine, cyclophosphamide, or other agents. Although there may be poor prognosis with the onset of autoimmune disease in patients with MDS, patients with a hematologic response to immunosuppressive therapy have prolonged survival compared with patient who do not. In this case, we noted that treatment with prednisone improved not only erythema nodosum and pericardial effusion, which may be related to an autoimmune manifestation, but also thrombocytopenia.

In summary, MDS may be associated with erythema nodosum with skin lesions that may be a result of autoimmune dysfunction. The understanding of the pathophysiology of MDS may contribute to explain the autoimmune manifestations and skin lesions in MDS.

\section{REFERENCES}

1) Avivi I, Rosenbaum H, Levy Y, Rowe J. Myelodysplastic syndrome and associated skin lesions: a review of the literature. Leuk Res 23:323-330, 1999

2) Castro M, Conn DL, Su WP, Garton JP. Rheumatic manifestations in myelodysplastic syndromes. J Rheumatol 18:721-727, 1991

3) Kuzmich PV, Ecker GA, Karsh J. Rheumatic manifestations in patients with myelodysplastic and myeloproliferative diseases. I Rheumatol 21:1649-1654, 1994

4) Nishie W, Kimura T, Kanagawa M. Sweet's syndrome evolved from recurrent erythema nodosum in a patient with myelodysplastic syndrome. J Dermatol 29:91-95, 2002

5) Ryan TJ. Cutaneous vasculitis: erythema nodosum. In: Rook $A$, Wilkinson DS, Ebling FJ, eds. Textbook of dermatology. p. 2196-2202, Oxford, Blackwell, 1998

6) Bohn S, Buchner S, Itin P. Erythema nodosum: 112 cases: epidemiology, clinical aspects and histopathology. Schweiz Med Wochenschr 127:1168-1176, 1997

7) Cribier B, Caille A, Heid E, Grosshans E. Erythema nodosum and associated diseases: a study of 129 cases. Int J Dermatol 37:667-672, 1998

8) Bonci A, di Lernia V, Merli F, Lo Scocco G. Erythema nodosum and Hodgkin's disease. Clin Exp Dermatol 26:408-411, 2001

9) Enright H, Jacob HS, Vercellotti G, Howe R, Belzer M, Miller W. Paraneoplastic autoimmune phenomena in patients with myelodysplastic syndromes: response to immunosuppressive therapy. $\mathrm{Br} J$ Haematol 91:403-408, 1995

10) Yoshida Y. Biology of myelodysplastic syndromes. Int J Cell Cloning 5:356-375, 1987

11) Mufti GJ, Figes A, Hamblin TJ, Oscier DG, Copplestone JA. Immunological abnormalities in myelodysplastic syndromes: 1. serum immunoglobulins and autoantibodies. Br J Haematol 63:143-147, 1986

12) Colombat $\mathrm{PH}$, Renoux $\mathrm{M}$, Lamagnere JP, Renoux G. Immunologic indices in myelodysplastic syndromes. Cancer 61:1075-1081, 1988

13) Karcher DS, Frost AR. The bone marrow in human immunodeficiency virus (HIV)-related disease: morphology and clinical correlation. Am J Clin Pathol 95:63-71, 1991 